\title{
Dietary Modification of Lower Urinary Tract Symptoms with Fermented Whey Product
}

\author{
Tiiu Kullisaar ${ }^{1}$, Silver Türk ${ }^{2}$, Margus Punab $^{3}$, Andre Veskioja ${ }^{4 *}$, Epp Songisepp ${ }^{4}$, Kersti Zilmer $^{1}$, \\ Mihkel Zilmer ${ }^{1}$ \\ ${ }^{1}$ Department of Biochemistry, Tartu University, Tartu, Estonia \\ ${ }^{2}$ Department of Microbiology, Tartu, Estonia \\ ${ }^{3}$ Andrology Center, Tartu University Hospital, Tartu, Estonia \\ ${ }^{4}$ Bio-Competence Centre of Healthy Dairy Products, Tartu, Estonia \\ Email: *aveskioja@gmail.com
}

Received March 11, 2013; revised April 12, 2013; accepted April 19, 2013

Copyright (C) 2013 Tiiu Kullisaar et al. This is an open access article distributed under the Creative Commons Attribution License, which permits unrestricted use, distribution, and reproduction in any medium, provided the original work is properly cited.

\begin{abstract}
After the age of 50, the prostate begins to increase in size. This is known as benign prostatic hypertrophy (BPH). Compression of urethra by enlarged prostate causes dribbling reduced force of the urinary stream, pain and occasional bleeding or infection. This causes difficulty in urinating and requires many men to get up several times during the night to urinate. The aim of our study was to evaluate an influence of new fermented whey based product (FWP) to several biochemical parameters and lower urinary tract symptoms (LUTS). Patients from the outpatient department of Andrology Center were randomized. This study involved the patients with minor to moderate LUTS, International Prostate Symptoms Score (IPSS) range 3 - 19 but not prostatitis (NIH-Chronic Prostatitis Symptom Index (NIH-CPSI) $<4$ and 2 -glass test negative for prostate inflammation and infection). Consumption of the FWP decreased the IPSS score in patients with moderate LUTS/BPH $(\mathrm{p}<0.001)$ whereas irritative and obstructive symptoms changed in parallel. There was a correlation between LUTS symptoms change and level of inflammation- and oxidative stress-related indices (blood high-sensitive C-reactive protein, hsCRP; glycated haemoglobin, HbAlc; oxidized low density lipoprotein, oxLDL; interleukine-10, IL-10 and 8-isoprostanes in the urine). Statistically significant changes in mentioned parameters occurred only in study group. Compression of urethra by enlarged prostate explains LUTS in BPH patients. Elevated oxidative stress $(\mathrm{OxS})$ intensifies peroxidation of cell membrane phospholipids. This generates 8-isoprostanes (8-EPI), the prostaglandin-like compounds that can exaggerate LUTS. 8-isoprostanes may cause constriction of bladder and urethra in nanomolar concentrations. Consuming the whey-based product fermented by special lactobacilli strains may improve LUTS as well as OxS and diminish LUTS-related inflammatory response.
\end{abstract}

Keywords: Lower Urinary Tract Symptoms (LUTS); Benign Prostatic Hypertrophy (BPH); Prostate; Fermented Whey Product (FWP); Oxidative Stress

\section{Introduction}

Lower urinary tract symptoms (LUTS) due to benign prostatic hypertrophy (BPH) affect approximately $25 \%$ of men worldwide after the age of 40 . When prostate enlarges, urethra becomes compressed. This causes difficulty in urinating and requires many men to get up several times during night to urinate including dribbling, reduced force of urinary stream, pain and occasional bleeding or infection. The prevalence of BPH reaches as high as $70 \%$ in the older men, although it is debatable whether such prevalence is reached in the $60-69$ or 80 -

"Corresponding author.
89 years old age group [1,2]. As reviewed by Parsons, 2010 [3] the epidemiologic correlates of BPH are serum dihydrotestosterone, obesity, elevated fasting glucose, diabetes, fat intake and inflammation that increase the risk while vegetables, regular limited alcohol consumption, exercise, and non-steroidal anti-inflammatory drugs (NSAIDs) decrease the risk. In an excellent paper, the combined effects of increased metabolism of testosterone into estradiol, increased insulin followed by increased leptin have been suggested as a vicious circle of causing estrogen overproduction that predisposes to not only BPH but also obesity, depression, type II diabetes and Alzheimer's disease [4]. The prostate enlargement ordi- 
nary risk factors are age, decreased sexuality and the level of androgens. Besides that, LUTS associates with oxidative stress (OxS). Elevation of OxS intensifies the peroxidation of cell membrane phospholipids followed by elevation of 8 -iso-prostanes ( 8 -EPI) that can cause LUTS. It has been demonstrated that antioxidative treatment reduced LUTS in BPH patients [5]. It can be partially explained by reducing 8 -EPI level in the bladder as it has been shown that 8-EPI can constrict bladder and urethra at nanomolar levels [6].

Fermentation of whey isolate by specific selected lactic acid bacteria (LAB) strains could be an interesting possibility to produce dairy functional food both with high nutrient density, bioquality (whey proteins have high bioquality) and alleviating effects on LUTS/BPH via reduction of OxS. The aim of was to evaluate an influence of original FWP both on OxS and inflammation-related indices and lower urinary tract symptoms

\section{Materials \& Methods}

Patients were selected from outpatient department of Andrology Center Tartu University Hospital. All of the patients were Caucasians. The patients gave written informed consent and the Tartu University Ethics Committee approved the study. All subjects enrolled met the inclusion and exclusion criteria and fulfilled International Prostate Symptom Score (IPSS) questionnaire. 58 patients of light and moderate LUTS/BPH were recruited into randomized double blind control group included clinical study. The inclusion/exclusion criteria were: $55 \pm$ 5 years of age, prostate specific antigen (PSA) $<4 \mathrm{ng} / \mathrm{ml}$, light to moderate LUTS (IPSS score 3 - 19), excluded prostatitis (NIH-Chronic Prostatitis Symptom Index (NIH-CPSI) $<4$ and 2-glass test negative for prostate inflammation and infection. All patients were randomized into two groups, control group $(n=19)$ consumed apple juice $200 \mathrm{~g}$ /day and study group $(\mathrm{n}=39)$ consumed $50 \mathrm{~g} /$ day of yellow fermented whey product during 2 weeks ( 7 patients consumed FWP, fulfilled IPSS but could not give blood samples).

Whey from semi-hard cheese production (cheese dust and fat removed) was heated to fermentation temperature and the strain L. plantarum MCC1 (DSM 23881) and the strain L. gasseri MCC2 (DSM 23882) were added. After addition, whey was fermented 20 hours and then pasteurized. The product was flavored with sea buckthorn jam, cooled, bottled and stored at $2^{\circ} \mathrm{C}-6^{\circ} \mathrm{C}$. Chemical/ physical parameters of whey and end product are presented in Table 1 (Estonian patent application EE 201100012 [7]).

Blood and urine samples were collected before and after treatment. Routine blood indices were assayed: lowdensity lipoprotein-cholesterol (LDL-Chol), high-density lipoprotein-cholesterol (HDL-Chol), glucose, HbA1c and Immunoglobulin E (IgE) using a certified Hitachi 912 automated analyzer in the local clinical laboratory (Roche
Table 1. Chemical parameters of whey and end product.

\begin{tabular}{ccc}
\hline Ingredient/parameter & Whey & Product \\
\hline Fat (\%) & $<0.1$ & $0.72 \pm 0.03$ \\
Protein (\%) & $0.66 \pm 0.10$ & $0.98 \pm 0.04$ \\
Carbohydrates (\%) & $4.07 \pm 0.59$ & $9.9 \pm 1.2$ \\
Dry matter (\%) & $5.37 \pm 0.27$ & $11.99 \pm 0.12$ \\
pH & $6.14 \pm 0.41$ & $3.99 \pm 0.14$ \\
\hline
\end{tabular}

Diagnostics LDL-Chol and HDL-Chol). Assayed blood $\mathrm{OxS}$ and inflammation-related indices were as follows: oxLDL, IL-10, hsCR and urine indices 8-EPI. OxLDL level was measured by using the Mercodia Oxidized LDL Enzyme-Linked Immunosorbent Assay (ELISA) kit, manufactured by Mercodia AB, Sweden. Plasma level of IL-10 was measured by an ELISA using a commercially available kit (Human IL-10 Immunoassay, catalogue number D1000B, R\&D Systems Inc. ${ }^{\circledR}$, Minneapolis, USA). To characterize systemic OxS the content of 8EPI was assayed in urine. This assay is a competitive enzyme-linked immunoassay for determining levels of 8-EPI in biological samples (BIOXYTECH 8-Isoprostane Assay, Cat. No. 21019; Oxis International, Inc., Portland, OR, USA). The hsCRP was determined by a latex particle-enhanced immunotur-bidimetric assay (Roche Diagnostics GmPh, Germany) with the automated analyzer Hitachi 912 [8].

\section{Results \& Discussion}

The parameters of used whey and fermented end-product are presented in Table 1. Changes in nutritional parameters (fat, protein, carbohydrates and dry matter content) caused by addition of sea buckthorn jam. The drop of $\mathrm{pH}$ is the result of biological activity of used strains and also addition of sea buckthorn jam. The inflammatory prostatitis and prostate cancer were excluded on the basis of investigation of prostate secretion and NIH-CPSI questionnaire. IPSS score (consists of 3 questions that measure type of irritation and 4 questions that measure obstruction), decreased in patients of moderate LUTS/BPH from $13.0 \pm 3.2$ to $8.6 \pm 3.5(\mathrm{p}<0.001)$ after the consumption of the FWP during two weeks. In the control group as well as in light LUTS/BPH group separately, the reduction of LUTS did not reach statistical significance (Table 2).

Consumption of the FWP reduced the level of 8-EPI (8-EPI is accepted as the gold standard marker of systemic lipid peroxidation as well as $\mathrm{OxS}$ ) in urine more than placebo (Table 3). The next well marker of OxSoxLDL (approved recently also by EFSA panel of NDA [9]) as well as the new marker of inflammation-hsCRP, decreased (Table 3) and had statistically significant correlation between each other (the Spearman correlation 
Table 2. International Prostate Symptoms Score (IPSS) in LUTS/BPH patients.

\begin{tabular}{ccccc}
\hline & \multicolumn{2}{c}{ Control group of patients $(\mathbf{n}=\mathbf{1 9})$} & \multicolumn{2}{c}{ Study group of patients $(\mathbf{n}=\mathbf{3 9})$} \\
\cline { 2 - 5 } & Before & After & Before & After \\
\hline Light LUTS/BPH & $\mathrm{n}=9$ & $3.5 \pm 1.6$ & $\mathrm{n}=13$ & $4.2 \pm 1.4$ \\
IPSS (3 - 7 point) & $5.0 \pm 1.1$ & & $5.0 \pm 1.3$ & \\
Medium LUTS/BPH & $\mathrm{n}=10$ & $11.6 \pm 3.5$ & $13.0 \pm 3.2$ & $8.6 \pm 3.5^{*}$ \\
IPSS (8 - 19 point) & $13.7 \pm 3.8$ & &
\end{tabular}

Table 3. Clinical/biochemical parameters of LUTS/BPH patients before and after consumption of special fermented whey product.

\begin{tabular}{|c|c|c|c|c|}
\hline & \multicolumn{2}{|c|}{ Control group of patients $(n=19)$} & \multicolumn{2}{|c|}{ Study group of patients $(n=32)$} \\
\hline & Before & After & Before & After \\
\hline hsCRP mg/L & $1.58 \pm 0.98$ & $1.92 \pm 1.38$ & $1.92 \pm 1.24$ & $1.55 \pm 0.98^{*}$ \\
\hline $\mathrm{HbA} 1 \mathrm{c} \%$ of total $\mathrm{Hb}$ & $5.88 \pm 0.27$ & $5.86 \pm 0.32$ & $5.87 \pm 0.35$ & $5.79 \pm 0.36^{* *}$ \\
\hline oxLDL U/L & $60.3 \pm 11.1$ & $61.0 \pm 9.2$ & $72.0 \pm 16$ & $70.0 \pm 16^{*}$ \\
\hline IL-10 pg/mL & $12.6 \pm 7.2$ & $11.4 \pm 5.7$ & $8.6 \pm 2.2$ & $10.0 \pm 2.8^{*}$ \\
\hline 8-isoprostanes $\mathrm{ng} / \mathrm{mmol}$ creatinine & $47.7 \pm 8.4$ & $44.6 \pm 10.2$ & $47.8 \pm 15.9$ & $37.2 \pm 10.1^{* * *}$ \\
\hline
\end{tabular}

${ }^{*} \mathrm{p}<0.05,{ }^{* *} \mathrm{p}<0.01,{ }^{* * *} \mathrm{p}<0.001$; There were no statistically significant differences between the patients groups in baseline values.

coefficient $\mathrm{r}=0.4, \mathrm{p}=0.017)$. In addition, the level of anti-inflammatory cytokine IL-10 increased and the percent of HbA1c decreased (Table 3). Antioxidant properties of hydrolyzed whey protein isolate have been reported earlier, as increase of intracellular glutathione and protection from oxidant-induced cell death were observed [10]. Hb glycation (level of HbAlc) is just a marker of the formation of advanced glycation/lipoxidation end products (AGE-s/ALE-s) that may both cause and amplify $\mathrm{OxS}$. Hb glycation is accepted as a gold standard marker for diabetes [11]. IL-10 levels in seminal fluid is associated with BPH $[12,13]$. HsCRP, glycation of $\mathrm{Hb}$, reduced glutathione (GSH), 8-EPI and oxLDL can be considered a cluster of markers very closely related to OxS. Cytokine IL-10 is accepted as an anti-inflammatory factor.

In our previous study, we observed an increase of urinary 8-EPI that correlated with urinary 8-OHdG (both are $\mathrm{OxS}$ markers) in inflammatory prostatitis patients [14]. In this study, we observed a decrease of 8-EPI and a strong correlation between the former and hsCRP in LUTS patients after consumption of FWP (Table 3). Increased value of hsCRP associated with LUTS/BPH may refer to tissue damage associated with higher level of $\mathrm{OxS}$.

LUTS may have several causes. BHP is characterized by hyperplasia of prostatic stromal and epithelial cells, resulting in the formation of large, fairly discrete nodules in the periurethral region of prostate. When sufficiently large, the nodules compress the urethral canal and cause a partial, or sometimes a virtually complete obstruction of the urethra, which interferes with the normal flow of urine. LUTS associates also with elevated OxS. Reactive oxygen species (ROS) can be produced by the cells other than leucocytes and OxS level is not always a marker of leucocytes per se, but rather a marker of tissue injury [15]. The iatrogenic bladder obstruction of rabbits resulted in a dramatic elevation of urinary 8-hydroxy-deoxyguanine $(8-\mathrm{OHdG})$, an oxidation product of DNA, concentration that could be reversed by $70 \%$ with antioxidant treatment [5]. In addition, the clinical arm of the same study demonstrated that antioxidant treatment reduced LUTS in BPH patients [5]. Obstructed tissues might generate ROS and release 8-EPI into urine via circulation or direct diffusion through urinary epithelium. The 8-EPI can affect lower urinary tract of rabbits in even nanomolar concentrations [6]. This suggests that 8 -isoprostanes could contribute to LUTS by contracting bladder and urethra. In addition, prostatic cells by themselves are able to secrete inflammatory mediators and finally to stimulate their own growth [16]. In summary, $\mathrm{BPH}$ is a possible cause elevated $\mathrm{OxS}$ and vice versa (Figure 10).

It is known that the patients with BPH and LUTS have a considerably higher prevalence of cardiovascular diseases (CVD) than the general population [17]. Increased level of systemic OxS in BPH patients poses risk for development of CVD (Figure 12). These risks may be partially explained by increased level of oxLDL, decreased level of anti-inflammatory IL-10, effect of 8-EPI on the thromboxanes receptors [2]. The causal relationships between BPH, its comorbid diseases and the ana- 


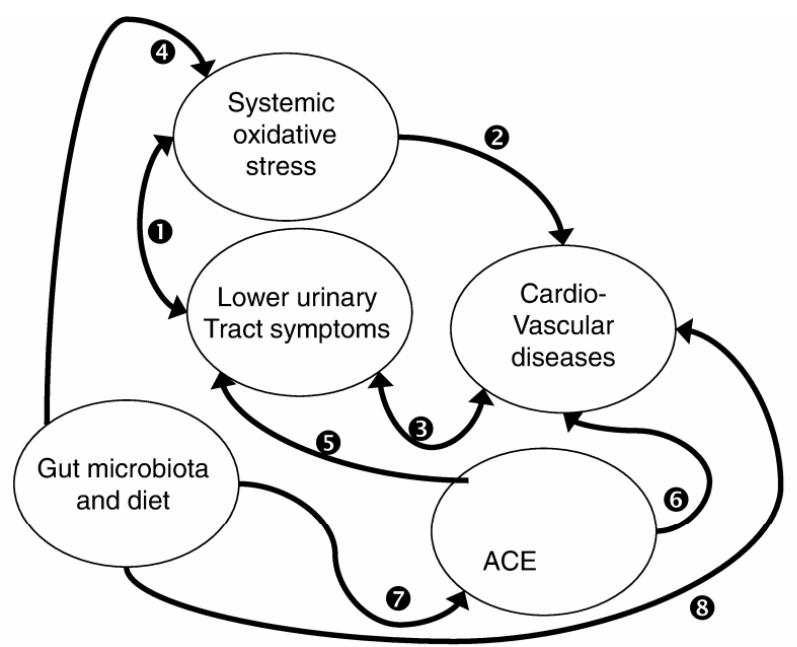

(1) $\mathrm{BPH}$ may contribute to systemic oxidative stress and vice versa.

2 Systemic oxidative stress may contribute to cardiovascular diseases.

3 The gist of this interaction is simple: urinary symptoms facilitate development of metabolic syndrome that facilitates development of cardiovascular diseases, and vice versa; this is a pathogenetic pathway is two-way.

(4) Principally, functional probiotic food may lower systemic oxidative stress but the evidence may be considered preliminary because no antioxidant probiotics with approved health claims are commercially available.

Figure 1. Schema of pathogenetic components possibly associated with whey product ameliorating LUTS.

lyzed markers (IL-10, hsCRP, 8-EPI, etc.) are not yet established. It is possible that CVD share harmful $\mathrm{OxS}$ as an ubiquitous pathogenetic component while metabolic syndrome may be a more specific etiologic factor common for both cardiovascular diseases and BPH [18].

However, a speculation that BPH may be causing nocturia-induced sleep disturbances, blood pressure variability, and increased sympathetic activity ([17]; Figure 13) is interesting indeed. LUTS may disturb everyday life, including circadian biological rhythms, to an extent that increases the risk for metabolic syndrome, which is, in turn, a well-established etiopathogenetic component of cardiovascular diseases [19].

Some data lends credence to hypothesis that some antioxidant and/or anti-inflammatory properties are due to cysteine and leucine, amino acids abundant in whey protein [20,21]. Interestingly, discovery of hydrogen sulfide synthesis from cysteine causing bladder relaxation may be a bit more concretely associated with LUTS [22]. Perhaps even more interestingly, they found out that bladder relaxation caused by sildenafil was mediated by $\mathrm{H}_{2} \mathrm{~S} . \mathrm{H}_{2} \mathrm{~S}$ contracts the bladder of rat [23] but relaxes human or trout bladders, instead [24,25]. There is a reason to believe that bladder-relaxing effect of $\mathrm{H}_{2} \mathrm{~S}$ is mediated by TRPA1 [26]. If TRPA1 pathway is involved, then TRPA1 levels in bladder [27], prostate [28], urethra [24], dorsal root ganglion peripheral nerve endings as well as dorsal horn of the spinal cord [29] may contribute to LUTS [30]. The whey proteins are valuable because of its high content of branched-chain essential amino acids isoleucine, leucine and valine [31] and sulfur-containing amino acid cysteine, the rate-limiting precursor of glutathione synthesis $[32,33]$. It is possible that the elevation of GSH in the system and tissues is the FWP's mechanism of action due to the decreased level of the redox ratio (GSSG/GSH) and this can suppress OxS and con sequent release of 8 -EPI. Additional explanation is that the whey protein promotes the growth of the beneficial bacteria, as was apparently the case in a study of a whey-based infant formula [34].

Finally, it is possible that the two lactobacilli strains used for fermentation contributed to benefit because the lactobacilli strains used for fermentation do have moderate antioxidative properties (Estonian patent application EE201100012 [7]). Our FWP may comprise special properties - antioxidant and anti-inflammatory-due to the products accumulated during whey protein fermentation, cooked lactobacilli (L. plantarum and L. delbrueckii) themselves, or both. Other studies showed that certain probiotic strains of lactic acid bacteria (LAB) and bifidobacteria may induce oral tolerance to $\beta$-lactoglobulin, restore aberrant protein transport and have specific effect on protein degradation in the intestinal mucosa [35]. It is also possible that the lactobacilli contributed to the bene- 
fit by modulating the gut microbiota. Introduction of new microorganisms into the gut may either decrease or increase the microbial diversity [36]. Composition of gut microbiota, and modulation thereof seems to have a profound ability to influence widely diverse facets of the hosts' physiology and metabolism [37]. For example, experimental yoghurt contains angiotensin converting enzyme (ACE) inhibiting peptides. The said ACE-inhibiting yoghurt has lowered blood pressure of rats ([38]; Figure 14 and (5). While synthetic ACE inhibitors are a widely known class of antihypertensive drugs (Figure 16), it may be at least reasonably speculated that ACE participates in the pathogenesis of $\mathrm{BPH}$ and that inhibition of ACE may contrib- ute to treatment of BPH or LUTS (Figure 10; [39]). And finally, a healthy diet contributes to cardiovascular health, of course (Figure $18 ;[40])$.

\section{Conclusion}

We can conclude that consuming the whey-based product fermented by special lactobacilli strains (L. plantarum $\mathrm{MCC} 1$ and $L$. gasseri MCC2) may improve LUTS as well as OxS status and diminish LUTS-related inflamematory response.

\section{Acknowledgements}

The research leading to these results is co-financed by the European Community's Regional Development Fund in the framework of the Competence Centre Programme of the Enterprise Estonia under project numbers EU22868; EU27789; EU28662; EU30002 of BioCompetence Centre Of Healthy Dairy Products (Tervisliku Piima Biotehnoloogiate Arenduskeskus OÜ).

\section{REFERENCES}

[1] J. T. Wei, E. Calhoun and S. J. Jacobsen, "Urologic Diseases in America Project: Benign Prostatic Hyperplasia," The Journal of Urology, Vol. 173, No. 4, 2005, pp. 12561261. doi:10.1097/01.ju.0000155709.37840.fe

[2] J. K. Parsons, J. Bergstrom, J. Silberstein and E. BarrettConnor, "Prevalence and Characteristics of Lower Urinary Tract Symptoms in Men Aged > or $=80$ Years," Urology, Vol. 72, No. 2, 2008, pp. 318-321. doi:10.1016/j.urology.2008.03.057

[3] J. K. Parsons, "Benign Prostatic Hyperplasia and Male Lower Urinary Tract Symptoms: Epidemiology and Risk Factors," Current Bladder Dysfunction Reports, Vol. 5, No. 4, 2010, pp. 212-218. doi:10.1007/s11884-010-0067-2

[4] G. Williams, "Aromatase Up-Regulation, Insulin and Raised Intracellular Oestrogens in Men, Induce Adiposity, Metabolic Syndrome and Prostate Disease, via Aberrant ER- $\alpha$ and GPER Signaling," Molecular and Cellular Endocrinology, Vol. 351, No. 2, 2012, pp. 269-278.

\section{doi:10.1016/j.mce.2011.12.017}

[5] S. Matsumoto, T. Hanai, T. Matsui, M. Oka, M. Tanaka and H. Uemura, "Eviprostat Suppresses Urinary Oxidative Stress in a Rabbit Model of Partial Bladder Outlet Obstruction and in Patients with Benign Prostatic Hyperplasia," Phytotherapy Research, Vol. 24, No. 2, 2010, pp. 301-303.

[6] T. Tarcan, M. B. Siroky, R. J. Krane and K. M. Azadzoi, "Isoprostane 8-epi PGF2alpha, a Product of Oxidative Stress, Is Synthesized in the Bladder and Causes Detrusor Smooth Muscle Contraction," Neurourology and Urodynamics, Vol. 19, No. 1, 2000, pp. 43-51. doi:10.1002/(SICI)1520-6777(2000)19:1<43::AID-NAU6 3.0.CO;2-C

[7] T. Kullisaar, M. Zilmer, E. Tammsaar, A. Veskioja, E. Songisepp, K. Zilmer, L. Bobrovski, K. Ehrlich, M. Rätsep, M. Punab, M. Vasar and M. Mikelsaar, "Isolated Strains of Microorganisms MCC1 DSM23881 and MCC2 DSM23882 and the Use Thereof," Applicant Bio-Competence Centre of Healthy Dairy Products.

[8] E. Pihl, K. Zilmer, T. Kullisaar, C. Kairane, A. Pulges and M. Zilmer, "High-Sensitive C-Reactive Protein Level and Oxidative Stress-Related Status in Former Athletes in Relation of Traditional Cardiovascular Risk Factors," Atherosclerosis, Vol. 171, No. 2, 2003, pp. 321-326. doi:10.1016/j.atherosclerosis.2003.08.015

[9] EFSA Panel on Dietetic Products, Nutrition and Allergies (NDA), "Scientific Opinion on the Substantiation of Health Claims Related to Polyphenols in Olive and Protection of LDL Particles from Oxidative Damage," EFSA Journal, Vol. 9, No. 4, 2011, pp. 2033-2058.

[10] K. D. Kent, W. J. Harper and J. A. Bomser, "Effect of Whey Protein Isolate on Intracellular Glutathione and Oxidant-Induced Cell Death in Human Prostate Epithelial Cells," Toxicology in Vitro, No. 17, 2003, pp. 27-33.

[11] S. Rahbar, "The Discovery of Glycated Hemoglobin: A Major Event in the Study of Nonenzymatic Chemistry in Biological Systems," Annals of the New York Academy of Sciences, Vol. 1043, 2005, pp. 9-19. doi:10.1196/annals.1333.002

[12] G. Penna, N. Mondaini, S. Amuchastegui, S. D. Innocenti, M. Carini, G. Giubilei, B. Fibbi, E. Colli, M. Maggi and L. Adorini, "Seminal Plasma Cytokines and Chemokines in Prostate Inflammation: Interleukin 8 as a Predictive Biomarker in Chronic Prostatitis/Chronic Pelvic Pain Syndrome and Benign Prostatic Hyperplasia," European Urology, Vol. 51, No. 2, 2007, pp. 524-533. doi:10.1016/j.eururo.2006.07.016

[13] K. H. Yoo, S. K. Kim, J. H. Chung and S. G. Chang, "Association of IL10, IL10RA, and IL10RB Polymorphisms with Benign Prostate Hyperplasia in Korean Population," Journal of Korean Medical Science, Vol. 26, No. 5, 2011, pp. 659-664. doi:10.3346/jkms.2011.26.5.659

[14] T. Kullisaar, S. Türk, M. Punab, P. Korrovits, K. Kisand, A. Rehema, K. Zilmer and M. Zilmer, "Oxidative Stress in Leucocytospermic Prostatitis Patients: Preliminary Results," Andrologia, Vol. 40, No. 3, 2008, pp. 161-172. doi:10.1111/j.1439-0272.2007.00816.x 
[15] A. M. Pontari, "Inflammation and Anti-Inflammatory Therapy in Chronic Prostatitis," Urology, Vol. 60, No. 6, 2002, pp. 29-33. doi:10.1016/S0090-4295(02)02381-6

[16] G. Robert, M. Salagierski, J. A. Schalken and A. de La Taille, "Inflammation and Benign Prostatic Hyperplasia: Cause or Consequence?" Progrès en Urologie, Vol. 20, No. 6, 2010, pp. 402-407. doi:10.1016/j.purol.2010.03.007

[17] O. F. Karatas, O. Bayrak, E. Cimentepe and D. Unal, “An Insidious Risk Factor for Cardiovascular Disease: Benign Prostatic Hyperplasia," International Journal of Cardiology, Vol. 144, No. 3, 2010, p. 452. doi:10.1016/j.ijcard.2009.03.099

[18] M. Gacci, L. Vignozzi, A. Sebastianelli, M. Salvi, C. Giannessi, C. De Nunzio, A. Tubaro, G. Corona, G. Rastrelli, R. Santi, G. Nesi, S. Serni, M. Carini and M. Maggi, "Metabolic Syndrome and Lower Urinary Tract Symptoms: The Role of Inflammation," Prostate Cancer and Prostatic Diseases, Vol. 16, No. 1, 2013, pp. 101-106. doi:10.1038/pcan.2012.44

[19] A. C. Reynolds, J. Dorrian, P. Y. Liu, H. P. Van Dongen, G. A. Wittert, L. J. Harmer and S. Banks, "Impact of Five Nights of Sleep Restriction on Glucose Metabolism, Leptin and Testosterone in Young Adult Men," PLoS One, Vol. 7, No. 7, 2012, Article ID: e41218. doi:10.1371/journal.pone.0041218

[20] W. G. Witham, K. A. Yester and K. R. McGaffin, “A High Leucine Diet Mitigates Cardiac Injury and Improves Survival after Acute Myocardial Infarction," Metabolism, Vol. 62, No. 2, 2013, pp. 290-302. doi:10.1016/j.metabol.2012.07.023

[21] S. Vasdev, J. Stuckless and V. Richardson, "Role of the Immune System in Hypertension: Modulation by Dietary Antioxidants," International Journal of Angiology, Vol. 20, No. 4, 2011, pp. 189-212. doi:10.1055/s-0031-1288941

[22] F. Fusco, R. D. di Villa Bianca, E. Mitidieri, G. Cirino, R. Sorrentino and V. Mirone, "Sildenafil Effect on the Human Bladder Involves the L-Cysteine/Hydrogen Sulfide Pathway: A Novel Mechanism of Action of Phosphodiesterase Type 5 Inhibitors," European Urology, Vol. 62, No. 6, 2012, pp. 1174-1180. doi:10.1016/j.eururo.2012.07.025

[23] R. Patacchini, P. Santicioli, S. Giuliani and C. A. Maggi, "Hydrogen Sulfide $\left(\mathrm{H}_{2} \mathrm{~S}\right)$ Stimulates Capsaicin-Sensitive Primary Afferent Neurons in the Rat Urinary Bladder," British Journal of Pharmacology, Vol. 142, No. 1, 2004, pp. 31-34. doi:10.1038/sj.bjp.0705764

[24] P. Weinhold, C. Gratzke, T. Streng, C. Stief, K. E. Andersson and P. Hedlund, "TRPA1 Receptor Induced Relaxation of the Human Urethra Involves TRPV1 and Cannabinoid Receptor Mediated Signals, and Cyclooxygenase Activation," The Journal of Urology, Vol. 183, No. 5, 2010, pp. 2070-2076. doi:10.1016/j.juro.2009.12.093

[25] R. A. Dombkowski, M. M. Doellman, S. K. Head and K. R. Olson, "Hydrogen Sulfide Mediates Hypoxia-Induced Relaxation of Trout Urinary Bladder Smooth Muscle," Journal of Experimental Biology, Vol. 209, 2006, pp.
3234-3240. doi:10.1242/jeb.02376

[26] C. Gratzke, T. Streng, E. Waldkirch, K. Sigl, C. Stief, K. E. Andersson and P. Hedlund, "Transient Receptor Potential A1 (TRPA1) Activity in the Human Urethra-Evidence for a Functional Role for TRPA1 in the Outflow Region," European Urology, Vol. 55, No. 3, 2009, pp. 696-704. doi:10.1016/j.eururo.2008.04.042

[27] S. Du, I. Araki, H. Kobayashi, H. Zakoji, N. Sawada and M. Takeda, "Differential Expression Profile of Cold (TRPA1) and Cool (TRPM8) Receptors in Human Urogenital Organs," Urology, Vol. 72, No. 2, 2008, pp. 450455. doi:10.1016/j.urology.2007.11.127

[28] C. Gratzke, P. Weinhold, O. Reich, M. Seitz, B. Schlenker, C. G. Stief, K. E. Andersson and P. Hedlund, "Transient Receptor Potential A1 and Cannabinoid Receptor Activity in Human Normal and Hyperplastic Prostate: Relation to Nerves and Interstitial Cells," European Urology, Vol. 57, No. 5, 2010, pp. 902-910. doi:10.1016/j.eururo.2009.08.019

[29] A. Pertovaara and A. Koivisto, "TRPA1 Ion Channel in the Spinal Dorsal Horn as a Therapeutic Target in Central Pain Hypersensitivity and Cutaneous Neurogenic Inflammation," European Journal of Pharmacology, Vol. 666, No. 1-3, 2011, pp. 1-4. doi:10.1016/j.ejphar.2011.05.027

[30] S. Türk and T. Kullisaar, "Are Prostatitis Symptoms Associated with an Isoprostane-Mediated Vicious Circle?" Medical Hypotheses, Vol. 77, No. 5, 2011, pp. 837-840. doi:10.1016/j.mehy.2011.07.050

[31] M. Pescuma, E. M. Hebert, F. Mozzi and G. F. de Valdes, "Functional Fermented Whey-Based Beverage Using Lactic Acid Bacteria," International Journal of Food Microbiology, Vol. 141, No. 1-2, 2010, pp. 73-81. doi:10.1016/j.ijfoodmicro.2010.04.011

[32] O. W. Griffith, "Biologic and Pharmacologic Regulation of Mammalian Glutathione Synthesis," Free Radical Biology and Medicine, Vol. 27, No. 9-10, 1999, pp. 922935. doi:10.1016/S0891-5849(99)00176-8

[33] M. Zilmer, U. Soomets, A. Rehema and Ü. Langel, "The Glutathione System as an Attractice Therapeutic Target," Letters in Drug Design \& Discovery, Vol. 2, 2005, pp. 121-127.

[34] J. M. Hascoët, C. Hubert, F. Rochat, H. Legagneur, S. Gaga, S. Emady-Azar and P. G Steenhout, "Effect of Formula Composition on the Development of Infant Gut Microbiota," Journal of Pediatric Gastroenterology and Nutrition, Vol. 52, No. 6, 2011, pp. 756-762. doi:10.1097/MPG.0b013e3182105850

[35] G. Prioult and I. Fliss, "Effect of Probiotic Bacteria and Maintenance of Oral Tolerance to Beta-Lactoglobulin in Gnotobiotic Mice," Clinical and Diagnostic Laboratory Immunology, Vol. 10, 2003, pp. 787-792.

[36] J. M. Uronis, J. C. Arthur, T. Keku, A. Fodor, I. M. Carroll, M. L. Cruz, C. B. Appleyard and C. Jobin, "Gut Microbial Diversity Is Reduced by the Probiotic VSL\#3 and Correlates with Decreased TNBS-Induced Colitis," Inflammatory Bowel Diseases, Vol. 17, No. 1, 2011, pp. 289-297. doi:10.1002/ibd.21366

[37] N. Larsen, F. K. Vogensen, F. W. van den Berg, D. S. 
Nielsen, A. S. Andreasen, B. K. Pedersen, W. A. Al-Soud, S. J. Sørensen, L. H. Hansen and M. Jakobsen, "Gut Microbiota in Human Adults with Type 2 Diabetes Differs from Non-Diabetic Adults," PLoS One, Vol. 5, No. 2, 2010, Article ID: e9085.

doi:10.1371/journal.pone.0009085

[38] L. Ramchandran and N. P. Shah, "Yogurt Can Beneficially Affect Blood Contributors of Cardiovascular Health Status in Hypertensive Rats," Journal of Food Science, Vol. 76, No. 4, 2011, pp. 131-136. doi:10.1111/j.1750-3841.2011.02127.x

[39] D. T. Dinh, A. G. Frauman, G. R. Somers, M. Ohishi, J.
Zhou, D. J. Casley, C. I. Johnston and M. E. Fabiani, "Evidence for Activation of the Renin-Angiotensin System in the Human Prostate: Increased Angiotensin II and Reduced AT(1) Receptor Expression in Benign Prostatic Hyperplasia," Journal of Pathology, Vol. 196, No. 2, 2002, pp. 213-219. doi:10.1002/path. 1021

[40] A. von Ruesten, S. Feller, M. M. Bergmann and H. Boeing, "Diet and Risk of Chronic Diseases: Results from the First 8 Years of Follow-Up in the EPIC-Potsdam Study," European Journal of Clinical Nutrition, Vol. 67, No. 4, 2013, pp. 412-419. doi:10.1038/ejen.2013.7

\section{List of Abbreviations}

8-EPI-8-isoprostanes

8-OHdG-8-hydroxy-deoxyguanosine

ACE-angiotensin-converting enzyme

AGE-advanced glycation end-product

ALE_-advanced lipoxidation end-product

$\mathrm{BPH}$-benign prostate hyperplasia

CVD_cardiovascular diseases

EFSA_-European Food Safety Agency

ELISA-Enzyme-Linked ImmunoSorbent Assay

EU-European Union

FWP - the fermented whey product

$\mathrm{GSH}$-reduced glutathione

GSSG-oxidized glutathione

HbA1c_-glycated hemoglobin

HDL_-high-density lipoprotein
hsCRP — high-sensitivity C-reactive protein

IL-10_interleukin 10

IPSS - international prostate symptom score

LAB-lactic acid bacteria

LDL-low-density lipoprotein

LUTS - lower urinary tract symptoms

NIH-CPSI-national institute of health chronic prostatitis symptom score

NSAID—non-steroidal anti-inflammatory drug

oxLDL_oxidized low-density lipoprotein

OxS-oxidative stress

PSA - prostate-specific antigen

ROS_reactive oxygen species

TRPA1-Transient Receptor Potential cation channel, subfamily A, member 1 